\title{
Multimodal Pilot Behavior in Multi-Axis Tracking Tasks with Time-Varying Motion Cueing Gains
}

\author{
P.M.T. Zaal* \\ San Jose State University Research Foundation, \\ San Jose, CA
}

\author{
D.M. Pool ${ }^{\dagger}$ \\ Delft University of Technology, \\ Delft, The Netherlands
}

In a large number of motion-base simulators, adaptive motion filters are utilized to maximize the use of the available motion envelope of the motion system. However, not much is known about how the time-varying characteristics of such adaptive filters affect pilots when performing manual aircraft control. This paper presents the results of a study investigating the effects of time-varying motion filter gains on pilot control behavior and performance. An experiment was performed in a motion-base simulator where participants performed a simultaneous roll and pitch tracking task, while the roll and/or pitch motion filter gains changed over time. Results indicate that performance increases over time with increasing motion gains. This increase is a result of a time-varying adaptation of pilots' equalization dynamics, characterized by increased visual and motion response gains and decreased visual lead time constants. Opposite trends are found for decreasing motion filter gains. Even though the trends in both controlled axes are found to be largely the same, effects are less significant in roll. In addition, results indicate minor cross-coupling effects between pitch and roll, where a cueing variation in one axis affects the behavior adopted in the other axis.

\section{Nomenclature}

$\begin{array}{ll}A & \text { sinusoid amplitude, deg } \\ e & \text { tracking error signal, deg } \\ f & \text { forcing function, deg } \\ G & \text { maximum rate of change, } \mathrm{s}^{-1} \\ H_{c} & \text { aircraft dynamics response } \\ H_{o l} & \text { open-loop response } \\ H_{p} & \text { pilot response } \\ j & \text { imaginary unit } \\ K_{f} & \text { motion cueing gain, }- \\ K_{m} & \text { pilot motion gain, }- \\ K_{s} & \text { stick gain, }- \\ K_{v} & \text { pilot visual gain, }- \\ k & \text { sinusoid index } \\ M_{1} & \text { time of maximum rate of change, } \mathrm{s} \\ N & \text { number of points } \\ n & \text { pilot remnant signal, deg } \\ n & \text { forcing function frequency integer factor } \\ s & \text { Laplace variable } \\ T_{A 1}, T_{A 2} & \text { amplitude filter time constants, } \mathrm{s} \\ T_{L} & \text { pilot lead time constants, } \mathrm{s} \\ T_{m} & \text { measurement time, s } \\ t & \text { time, } \mathrm{s} \\ u & \text { pilot control signal, deg }\end{array}$

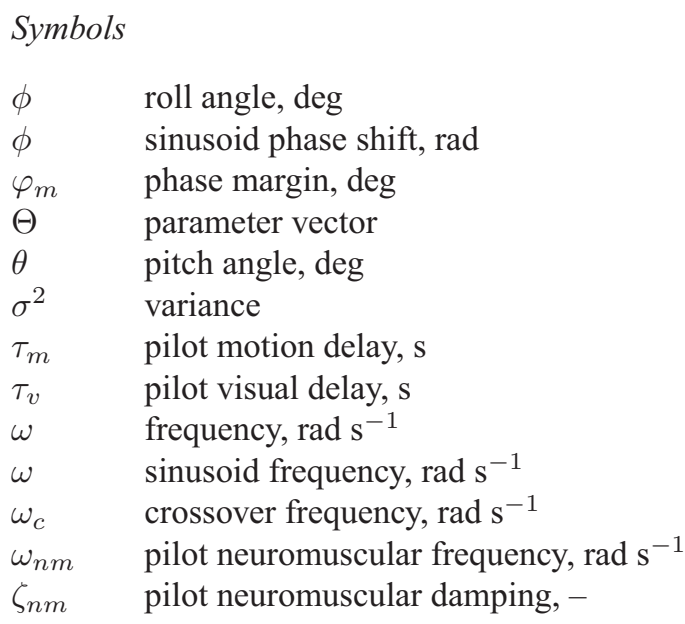

Subscripts

$\begin{array}{ll}1 & \text { initial value } \\ 2 & \text { final value } \\ d & \text { disturbance } \\ t & \text { target } \\ \phi & \text { roll } \\ \theta & \text { pitch }\end{array}$

\footnotetext{
* Research Associate, Human Systems Integration Division, NASA Ames Research Center, Moffett Field, CA, 94035; peter.m.t.zaal@nasa.gov. Member AIAA.

${ }^{\dagger}$ Assistant Professor, Control and Simulation Division, Faculty of Aerospace Engineering, P.O. Box 5058, 2600GB Delft, The Netherlands; d.m.pool@tudelft.nl. Member AIAA.
} 


\section{Introduction}

Much of the available knowledge on manual control behavior is restricted to stationary and time-invariant tasks, where the human controller is considered as a quasi-linear, but also a stationary and time-invariant controller. ${ }^{1-3}$ In many situations, however, it is the human controller's adaptation to changes in important task variables that is actually of interest. ${ }^{4}$ An example of a situation where such time-varying control behavior occurs, and for which interest has been steadily increasing, ${ }^{5-9}$ is one where a pilot is faced with a sudden change in aircraft dynamics. Such a change in the controlled system dynamics can occur, for instance, in the case of a damaged aircraft and typically requires significant adaptation of the pilot's control behavior to retain stability and satisfactory performance. For studying such time-varying characteristics of manual control behavior, progress has been made recently in the modeling and identification of time-varying pilot control behavior. ${ }^{5-9}$

Another situation in which time-varying manual control behavior may be induced is in flight simulators with adaptive motion cueing. To make more efficient use of a flight simulator's motion workspace, it is common to apply motion drive algorithms with time-varying dynamics. ${ }^{10-15}$ Such adaptive motion drive algorithms often introduce motion cueing gains that vary over time. The value of these gains is typically calculated by an online steepest-descent optimization scheme that continuously attempts to keep the motion cueing gains as high as possible, but which quickly reduces them when the simulator approaches the limits of its motion workspace. ${ }^{10,13}$

Earlier experiments have shown that in single-axis tracking tasks with simulator motion feedback, multimodal pilot control behavior is highly sensitive to variations in the gains and break frequencies of linear time-invariant motion filters. ${ }^{16-18}$ These studies show that with decreasing motion cueing gains, pilots rely less on the supplied motion feedback, which typically results in degraded task performance. It is currently unknown if similar adaptation of manual control behavior also occurs for time-varying motion cueing gains, and if so, how quickly this adaptation occurs. Furthermore, in situations where pilots are actively controlling multiple degrees-of-freedom of an aircraft, such possible behavioral adaptation is not necessarily restricted to the degree-of-freedom for which the motion cueing gain was changed. The change of a motion cueing gain in a single degree of freedom may alter the overall sensation of motion and result in behavioral adaptation in degrees-of-freedom for which no change was actually present. Such cross-coupling effects have also not been explicitly studied in tracking tasks.

For these reasons, this paper considers the adaptation of pilot tracking behavior in a combined pitch and roll tracking task with time-varying motion cueing gains. An experiment, performed in the SIMONA Research Simulator (SRS) at Delft University of Technology (DUT), is described in which the motion cueing gains in a dual-axis tracking task were either constant, or varied over time according to a sigmoid function. Both decreasing and increasing motion cueing gains were considered. The presence of time-varying motion cueing gains in the pitch and roll axes was varied independently to allow for detection of the independent direct effects, but also of possible cross-coupling effects, on the adopted control behavior. Pilot control behavior in this dual-axis tracking task is modeled using a multimodal pilot model with time-varying parameters, thereby expanding the time-varying modeling and identification method applied in Ref. 8.

This paper is structured as follows. First, Section II provides the details of the considered combined pitch and roll tracking task, as well as the time-varying multimodal pilot model that is used for analyzing the behavior adopted in this task. The experimental methods and hypotheses are described in Section III. The experimental results are then presented in Section IV. The paper ends with a discussion and the main conclusions.

\section{Time-Varying Multi-Axis Control Task}

Fig. 1 depicts the multi-axis control task performed in the SIMONA Research Simulator (SRS). A pilot simultaneously controls the aircraft's roll and pitch attitude, $\phi$ and $\theta$. To allow for accurate identification of pilot visual and vestibular responses in both pitch and roll, a simultaneous target-following and disturbance-rejection task is performed in each axis. ${ }^{19,20}$ The aircraft roll and pitch dynamics are perturbed by disturbance forcing functions $f_{d \phi}$ and $f_{d \theta}$. The errors between the aircraft roll and pitch angles and the corresponding target forcing functions $f_{t \phi}$ and $f_{t \theta}$ are presented on a two-axis compensatory display, similar to a attitude indicator. The pilot's task is to follow the roll and pitch targets by keeping the roll and pitch errors $e_{\phi}$ and $e_{\theta}$ as small as possible at all times. In addition to the visual stimuli presented on the PFD, rotational roll and pitch motion stimuli are provided by the simulator motion system. The presented motion stimuli are attenuated by time-varying motion gains $K_{f \phi}$ and $K_{f \theta}$ in the roll and pitch axes, respectively. Finally, the pilot makes control inputs $u_{\phi}$ and $u_{\theta}$ using a joystick with roll and pitch gains $K_{s \phi}$ and $K_{s \theta}$, closing the control loop. To allow for isolating the effects of the applied motion cueing on pilot tracking behavior, the pitch and roll tracking tasks were chosen to be independent (no cross-coupling in the controlled dynamics) and to have 


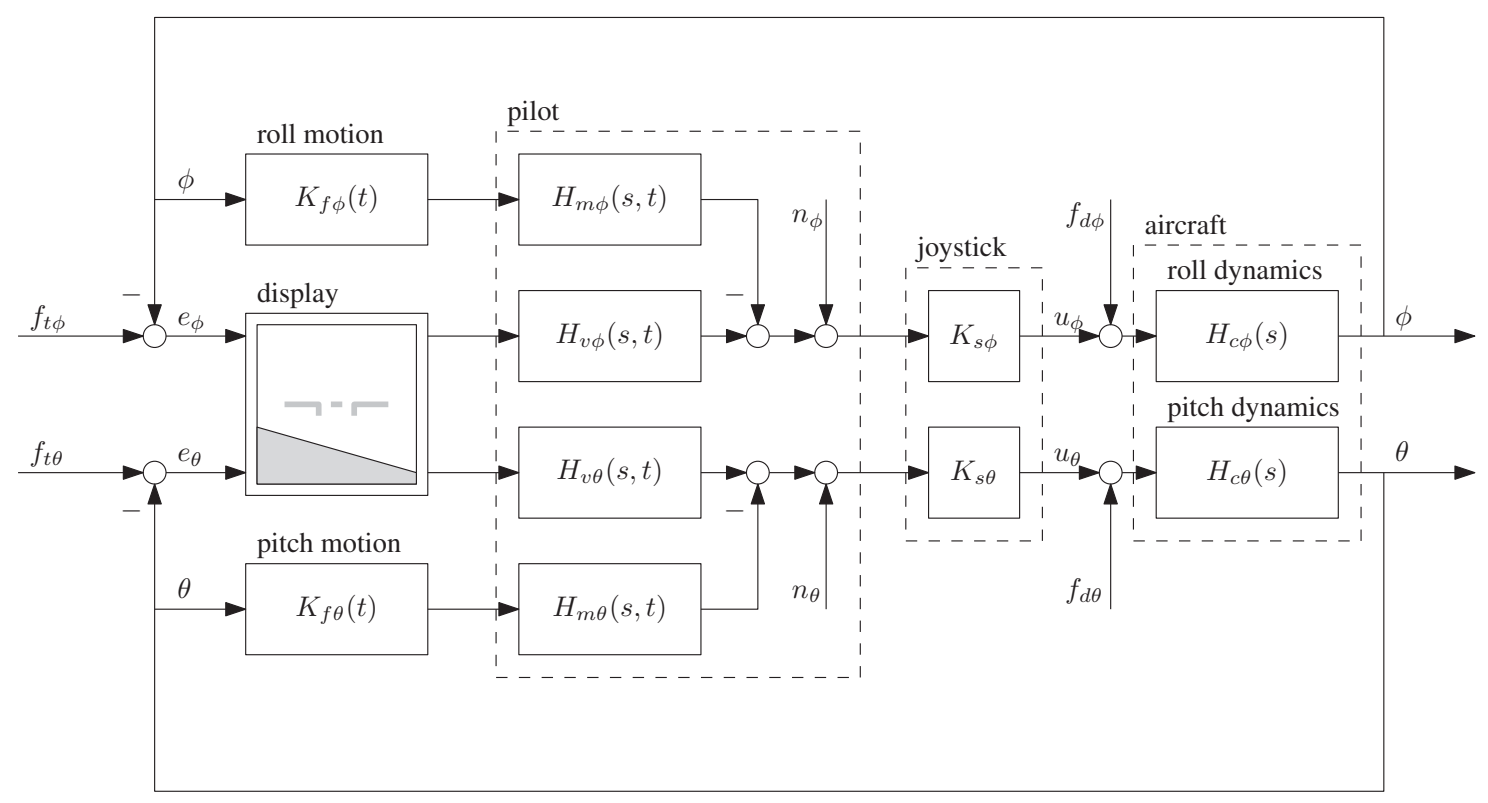

Figure 1. Multi-axis multi-loop control task.

identical controlled element dynamics and forcing function signals. The following sections describe the controlled dynamics, forcing functions, time-varying motion and pilot model for this task.

\section{II.A. Controlled Aircraft Dynamics}

The aircraft roll and pitch dynamics are defined by $H_{c \phi}(s)$ and $H_{c \theta}(s)$, respectively (see Fig. 1). The dynamics in both axes are assumed to be equal and there is no cross coupling; that is, a roll control input only affects the roll attitude and a pitch control input only affects the pitch attitude. The dynamics in both axes are defined by the following second-order transfer function:

$$
H_{c \phi}(s)=H_{c \theta}(s)=\frac{30.0}{s^{2}+0.2 s}
$$

with $s$ the Laplace variable. This controlled element was also considered in an earlier experiment that investigated time-varying pilot control behavior. ${ }^{8}$ With a break frequency of $0.2 \mathrm{rad} / \mathrm{s}$, these controlled element dynamics approximate a double integrator system $\left(1 / s^{2}\right)$ in the frequency range where the pilot-vehicle system crossover frequency is expected to be (2.5-5 rad/s). ${ }^{21}$ This implies that pilots will need to perform lead equalization. It has been shown that for such controlled elements, pilot tracking behavior is significantly affected by the presence, and quality, of simulator motion feedback. ${ }^{22}$

\section{II.B. Forcing Functions}

The target and disturbance forcing functions are sum-of-sine signals defined by:

$$
f_{d, t}(t)=\sum_{k=1}^{N_{d, t}} A_{d, t}(k) \sin \left[\omega_{d, t}(k) t+\phi_{d, t}(k)\right]
$$

where the subscripts $d$ and $t$ indicate the disturbance or target forcing function, respectively. In Eq. (2), $A(k), \omega(k)$ and $\phi(k)$ indicate the amplitude, frequency and phase of the $k^{\text {th }}$ sine in $f_{d}$ or $f_{t} . N_{d, t}$ indicates the number of sine waves. Both $f_{d}$ and $f_{t}$ consist of 10 individual sinusoids with a different amplitude, frequency and phase. The target forcing functions in roll and pitch have equivalent frequency and amplitude distributions, however, the phase distributions are different to give the forcing functions a different appearance in each axis. The same holds for the disturbance forcing functions. A summary of all forcing function properties can be found in Table 1.

The frequencies of the sinusoids, $\omega_{d}(k)$ and $\omega_{t}(k)$, were all integer multiples of the measurement time base frequency, $\omega_{m}=2 \pi / T_{m}=0.0767 \mathrm{rad} / \mathrm{s}$, where $T_{m}=81.92 \mathrm{~s}$ is the measurement time (see Section III). The chosen 
Table 1. Forcing function properties.

\begin{tabular}{rllllrllll}
\hline \hline \multicolumn{9}{c}{ disturbance, $f_{d}$} \\
\hline$n_{d},-$ & $\omega_{d}, \mathrm{rad} \mathrm{s}^{-1}$ & $A_{d}, \mathrm{deg}$ & $\phi_{d \phi}, \mathrm{rad}$ & $\phi_{d \theta}, \mathrm{rad}$ & $n_{t},-$ & $\omega_{t}, \mathrm{rad} \mathrm{s}^{-1}$ & $A_{t}$, deg & $\phi_{t \phi}, \mathrm{rad}$ & $\phi_{t \theta}, \mathrm{rad}$ \\
\hline 5 & 0.383 & 0.009 & 2.746 & 2.875 & 6 & 0.460 & 1.657 & 5.594 & 0.214 \\
11 & 0.844 & 0.029 & 2.147 & 3.010 & 13 & 0.997 & 1.159 & 5.061 & 0.101 \\
23 & 1.764 & 0.063 & 3.942 & 6.036 & 27 & 2.071 & 0.523 & 1.123 & 3.008 \\
37 & 2.838 & 0.082 & 0.097 & 2.846 & 41 & 3.145 & 0.281 & 2.477 & 6.058 \\
51 & 3.912 & 0.095 & 2.335 & 1.078 & 53 & 4.065 & 0.189 & 4.309 & 4.270 \\
71 & 5.446 & 0.112 & 1.392 & 3.672 & 73 & 5.599 & 0.117 & 0.364 & 0.567 \\
101 & 7.747 & 0.141 & 5.297 & 4.709 & 103 & 7.900 & 0.074 & 1.746 & 1.593 \\
137 & 10.508 & 0.188 & 5.818 & 0.617 & 139 & 10.661 & 0.054 & 5.795 & 3.778 \\
171 & 13.116 & 0.244 & 2.849 & 4.600 & 194 & 14.880 & 0.042 & 3.467 & 1.473 \\
226 & 17.334 & 0.361 & 1.820 & 5.383 & 229 & 17.564 & 0.039 & 5.665 & 2.253 \\
\hline \hline
\end{tabular}

integer multiples were used in a number of earlier experiments and ensured that the ten sinusoid frequencies in each signal covered the frequency range of human control with regular intervals on a logarithmic scale. $6,8,20,23,24$

A second-order low-pass filter was used to determine the amplitudes of the individual sines for both the target and the disturbance forcing function:

$$
A_{d, t}(k)=\left|\frac{1+T_{A 1} j \omega_{d, t}(k)}{1+T_{A 2} j \omega_{d, t}(k)}\right|^{2}
$$

with $T_{A 1}=0.1 \mathrm{~s}$ and $T_{A 2}=0.8 \mathrm{~s}$. This second-order filter reduces the magnitude of the amplitudes at the higher frequencies, yielding a tracking task that is not overly difficult. The amplitude distributions $A_{d}(k)$ and $A_{t}(k)$ were scaled to attain variances for $f_{d}$ and $f_{t}$ of $2.25 \mathrm{deg}^{2}$ and $0.56 \mathrm{deg}^{2}$, respectively; that is, the target had only $25 \%$ of the power of the disturbance. This relative strength of the target and disturbance signals and the amplitude variation determined by Eq. (3) were successfully applied for measuring pilot tracking behavior in earlier experiments. ${ }^{23,24}$

To determine the forcing function phase distributions, a large number of random sets of phases were generated. Four sets of phases - for the target and disturbance signals in the pitch and roll axes - were chosen that yielded signals with an approximately Gaussian distribution and an average crest factor, as well as an average crest factor for the first and second derivatives of the signals, as proposed in Ref. 25.

The disturbance signal amplitudes and phases were prefiltered with the inverse of the aircraft dynamics, as the disturbance signal is attenuated by the controlled aircraft dynamics (Fig. 1). This ensured that $f_{d}$ induced the desired low-pass disturbance of the roll or pitch attitude after passing through the controlled dynamics. It should be noted that Table 1 lists these prefiltered disturbance signal amplitudes and phases.

\section{II.C. Time-Varying Motion Gains}

The parameter function used to vary the motion filter gains over time is the generalized logistic function, a widely-used sigmoid function. Sigmoid functions are characterized by their S-shape, describing the transition from an initial value to a final value. This parameter function was also used in a previous study on timevarying pilot control behavior. ${ }^{8}$ The generalized logistic function as a function of time $t$ for the roll motion gain is defined by:

$$
K_{f \phi}(t)=K_{f \phi 1}+\frac{K_{f \phi 2}-K_{f \phi 1}}{1+e^{-G_{f}\left(t-M_{f}\right)}}
$$

This generalized logistic function defines a smooth

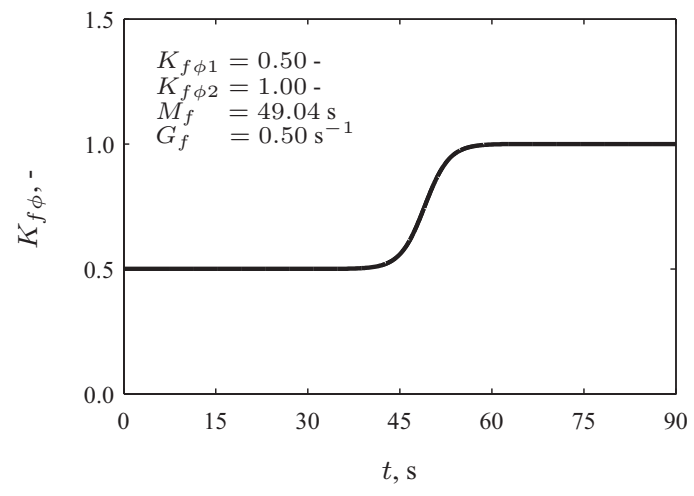

Figure 2. Time-varying motion filter gain. transition from an initial motion gain $K_{f \phi 1}$ to a final motion gain value $K_{f \phi 2}$, where the time this transition occurs is defined by $M_{f}$ and its maximum rate of change by $G_{f}$. The motion gain in the pitch axis $K_{f \theta}(t)$ is defined equivalently to Eq. (4). For the experiment discussed in this paper, motion gain transitions from 0.5 to 1.0 and 1.0 to 0.5 are considered. Fig. 2 depicts the roll motion gain for $K_{f \phi 1}=0.5, K_{f \phi 2}=1.0, M_{f}=49.04 \mathrm{~s}$ and $G_{f}=0.5 \mathrm{~s}^{-1}$. 


\section{II.D. Pilot Model}

The use of two forcing functions in both the roll and pitch axes allows for the identification of two linear pilot visual response functions $H_{v \phi}$ and $H_{v \theta}$, and two linear pilot motion response functions $H_{m \phi}$ and $H_{m \theta}$ (Fig. 1). Nonlinear pilot control behavior and experiment measurement noise are captured by the remnant signals $n_{\phi}$ and $n_{\theta}$.

Based on previous experiments with varying motion stimuli and the fact that pilots adapt their equalization dynamics such that the pilot-aircraft open-loop system approximates single-integrator dynamics in the crossover region, ${ }^{1}$ the following model can be defined for the pilot visual response in the roll axis:

$$
H_{v \phi}(s, t)=K_{v \phi}(t)\left(1+T_{L \phi}(t) s\right) e^{-s \tau_{v \phi}} \frac{\omega_{n m \phi}^{2}(t)}{\omega_{n m \phi}^{2}(t)+2 \zeta_{n m \phi}(t) \omega_{n m \phi}(t) s+s^{2}}
$$

Note that the model for the pilot visual response given by Eq. (5) is equivalent to the precision model ${ }^{21}$ for the controlled dynamics given by Eq. (1), but now includes time-varying parameters $K_{v \phi}, T_{L \phi}, \omega_{n m \phi}$, and $\zeta_{n m \phi}$. The corresponding model for the pilot motion response is defined by:

$$
H_{m \phi}(s, t)=s K_{m \phi}(t) e^{-s \tau_{m \phi}} \frac{\omega_{n m \phi}^{2}(t)}{\omega_{n m \phi}^{2}(t)+2 \zeta_{n m \phi}(t) \omega_{n m \phi}(t) s+s^{2}}
$$

In these time-varying pilot transfer functions, the pilot equalization dynamics are defined by the pilot visual gain $K_{v \phi}$, lead time constant $T_{L \phi}$, and pilot motion gain $K_{m \phi}$. The neuromuscular dynamics are defined by the neuromuscular damping ratio $\zeta_{n m \phi}$ and neuromuscular frequency $\omega_{n m \phi}$. These five time-varying pilot model parameters are all defined by time-dependent generalized logistic functions equivalent to Eq. (4). To reduce the number of model parameters, the time-of-maximum-growth and maximum-rate-of-change parameters, $M_{p \phi}$ and $G_{p \phi}$, are equal for all five time-varying parameters of the pilot model in the roll axis. In addition, the pilot visual and motion delays in each axis, defined by $\tau_{v \phi}$ and $\tau_{m \phi}$, are assumed to remain constant over time. The time-varying transfer functions in the pitch axis, $H_{v \theta}(s, t)$ and $H_{m \theta}(s, t)$, are defined equivalently to Equations 5 and 6 . The resulting two-axis multimodal pilot model has a total of 14 parameters in each axis that are used to quantify time-varying control behavior in this experiment:

$$
\begin{gathered}
\Theta_{\phi}=\left[\begin{array}{llll}
K_{v \phi 1} & K_{v \phi 2} T_{L \phi 1} T_{L \phi 2} K_{m \phi 1} & K_{m \phi 2} \zeta_{n m \phi 1} \zeta_{n m \phi 2} \omega_{n m \phi 1} \omega_{n m \phi 2} M_{p \phi} G_{p \phi} \tau_{v \phi} \tau_{m \phi}
\end{array}\right] \\
\Theta_{\theta}=\left[\begin{array}{lll}
K_{v \theta 1} & K_{v \theta 2} T_{L \theta 1} T_{L \theta 2} K_{m \theta 1} K_{m \theta 2} \zeta_{n m \theta 1} \zeta_{n m \theta 2} \omega_{n m \theta 1} \omega_{n m \theta 2} M_{p \theta} G_{p \theta} \tau_{v \theta} \tau_{m \theta}
\end{array}\right]
\end{gathered}
$$

The pilot models are fit to experiment data by estimating the parameters using the time-domain maximum likelihood estimation (MLE) procedure discussed in Ref. 8.

\section{II.E. Open-Loop Dynamics}

In the frequency domain, pilot performance in attenuating the target and disturbance signals is determined by the crossover frequencies and phase margins of the target and disturbance open-loop dynamics, respectively. ${ }^{26}$ Using the control task diagram in Fig. 1 and the pilot response functions in Equations 5 and 6, the time-varying disturbance and target open-loop responses for the roll axis are given by:

$$
\begin{gathered}
H_{o l, d \phi}(s, t)=\left[H_{v \phi}(s, t)+K_{f \phi}(t) H_{m \phi}(s, t)\right] K_{s \phi} H_{c \phi}(s) \\
H_{o l, t \phi}(s, t)=\frac{H_{v \phi}(s, t) K_{s \phi} H_{c \phi}(s)}{1+K_{f \phi}(t) H_{m \phi}(s, t) K_{s \phi} H_{c \phi}(s)}
\end{gathered}
$$

The disturbance and target crossover frequencies, $\omega_{c, d}(t)$ and $\omega_{c, t}(t)$, are the frequencies where the magnitude of the disturbance and target open-loop responses have a magnitude of 1.0. The corresponding phase margins, $\varphi_{m, d}(t)$ and $\varphi_{m, t}(t)$, are the phase differences from -180 degrees at these crossover frequencies. The open-loop characteristics in the pitch axis are determined equivalently. 


\section{Experiment}

An experiment was performed on the SRS at DUT to determine the effects of time-varying roll and pitch motion filter gains on multimodal pilot control behavior in the combined roll and pitch control task described in Section II. This section describes the main experimental methods, as well as the experiment hypotheses.

\section{III.A. Method}

\section{III.A.1. Apparatus and Cueing}

The experiment was performed on the SRS, see Fig. 3. Participants were seated in the right pilot seat in the simulator cabin and used a Moog FCS Ecol-8000 electrical sidestick for giving pitch and roll control inputs. The characteristics of this control loaded sidestick could be fully adjusted and were set to a linear force-displacement characteristic with a stiffness of $1.5 \mathrm{~N} / \mathrm{deg}$ with no added mass, damping, or breakout force. For both axes, control inputs were limited to \pm 15 deg. The joystick output scaling gains for the roll and pitch axes - as indicated in Fig. 1 - were set to $K_{s \phi}=K_{s \theta}=0.08$.

The SRS has a hydraulic six degree-of-freedom hexapod motion system, which was used to supply the participants with pitch and roll motion feedback corresponding to the outputs of the controlled pitch and roll dynamics, $\theta$ and $\phi$ in Fig. 1, respectively. These pitch and roll motion cues were presented around a center of rotation that was aligned with the subjects' vertical body axis and located $0.7 \mathrm{~m}$ below the right pilot's design head position. No other motion cueing except for this combined rotational pitch and roll motion was performed. The time delay of the SRS motion cues is $\tau_{m s} \approx 30 \mathrm{~ms}$ in all axes. ${ }^{27}$ To ensure participants did not hear any sound coming from the motion-base actuators during the experiment, they were asked to wear noise-canceling headphones.

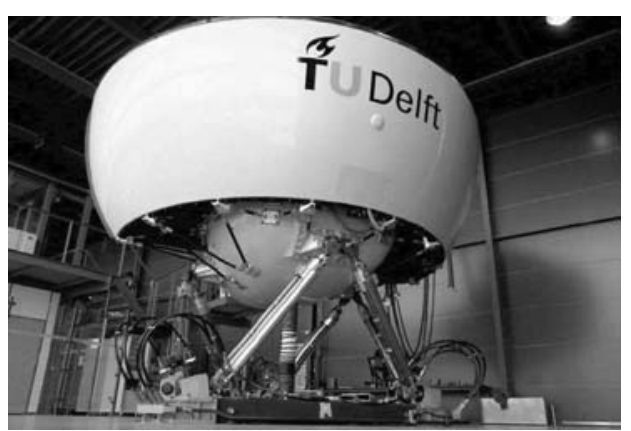

Figure 3. The SIMONA Research Simulator.

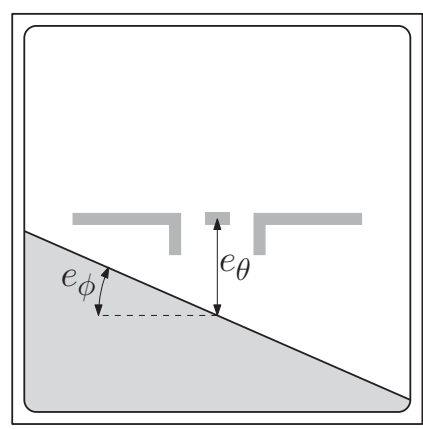

Figure 4. Primary flight display.

In addition to the supplied pitch and roll motion cues, the compensatory display (Fig. 4) was shown on a display directly in front of the participants. No other visual cues, for instance from the outside visual system, were provided. As indicated in Fig. 4, this compensatory visual display presented the participants with only the pitch and roll tracking errors, $e_{\theta}$ and $e_{\phi}$, respectively. The time delay of the image generation on the display has been measured to be in the order of $\tau_{d s} \approx 25 \mathrm{~ms}^{28}$

\section{III.A.2. Independent Variables}

Three independent variables, with two levels each, were varied in the experiment. The roll-axis motion gain was either constant or time-varying, the pitch-axis motion gain was either constant or time-varying, and the initial motion gain was either low or high. In the remainder of this paper, these three independent variables (factors) are referred to using the symbols "RS", "PS", and "SG", respectively. As explained in Section II.C, the low and high motion gain values considered were 0.5 and 1.0, respectively. For the experiment, a full-factorial design was implemented, resulting in the total of eight experimental conditions listed in Table 2. Conditions 1 to 4 -referred to with the symbols $\mathrm{C} 1$ to $\mathrm{C} 4-$ had a low initial motion gain of 0.5 . Conditions 5 to 8 (C5-C8) had a high initial motion gain of 1.0. In the conditions that considered a time-varying motion gain in the roll or pitch axes, the motion gain changed from 0.5 to 1.0 or from 1.0 to 0.5 . In these conditions, the sigmoid function given by Eq. (4) was used to change the values of $K_{f \phi}$ and $K_{f \theta}$ over time. For both axes, the time of maximum rate $M_{f}$ of the time-varying motion gain was set to $49.04 \mathrm{~s}$ (the middle of the $81.92 \mathrm{~s}$ measurement window, see Section III.A.4) and the maximum rate $G_{f}$ was set to 0.5 . 
Table 2. Experiment conditions.

\begin{tabular}{lcll}
\hline \hline Cond. & Factor (Level) & Roll gain, $K_{f \phi}$ & Pitch gain, $K_{f \theta}$ \\
\hline C1 & SG(1), RS(1), PS(1) & 0.5 & 0.5 \\
C2 & SG(1), RS(2), PS(1) & $0.5 \rightarrow 1.0$ & 0.5 \\
C3 & SG(1), RS(1), PS(2) & 0.5 & $0.5 \rightarrow 1.0$ \\
C4 & SG(1), RS(2), PS(2) & $0.5 \rightarrow 1.0$ & $0.5 \rightarrow 1.0$ \\
\hline C5 & SG(2), RS(1), PS(1) & 1.0 & 1.0 \\
C6 & SG(2), RS(2), PS(1) & $1.0 \rightarrow 0.5$ & 1.0 \\
C7 & SG(2), RS(1), PS(2) & 1.0 & $1.0 \rightarrow 0.5$ \\
C8 & SG(2), RS(2), PS(2) & $1.0 \rightarrow 0.5$ & $1.0 \rightarrow 0.5$ \\
\hline \hline
\end{tabular}

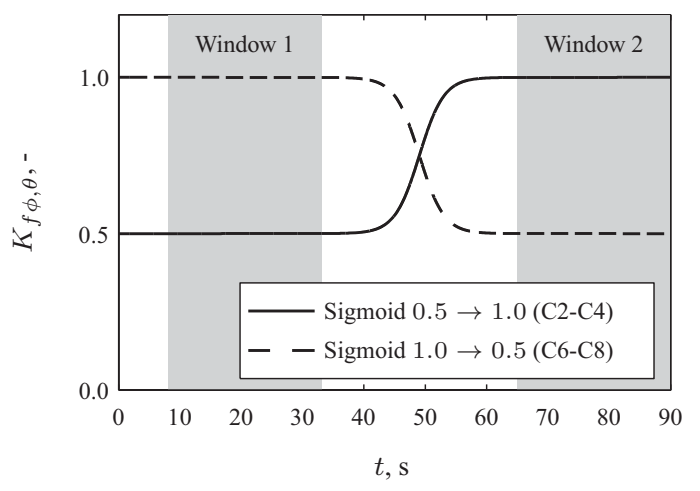

Figure 5. Sigmoid windows used for variance calculations.

\section{III.A.3. Participants and Instructions}

The experiment was performed by eight participants. All participants were students or staff members at the Control \& Simulation Division of the Faculty of Aerospace Engineering of DUT and had extensive experience with manual tracking tasks from earlier experiments. Two participants were active pilots on DUT's Cessna Citation II laboratory aircraft, and three others were active general aviation pilots.

Before the start of the experiment, participants received a briefing that informed them of the background of the study and of their task during the experiment. Their task was described as the simultaneous minimization of the pitch and roll tracking errors shown on the visual display (Fig. 4). No suggestions for a strategy for tracking two axes simultaneously were included in the briefing.

Participants were informed of the fact that they would be performing the combined pitch and roll tracking task with eight different motion cueing settings, as well as that for some experimental conditions the motion cueing would change during the tracking runs. They were made aware of the fact that motion cueing in the pitch and roll axes might be different for part of the experimental conditions. No further details of the experimental conditions and evaluated motion cueing settings (e.g., motion gain values, time-varying functions) were, however, shared with the participants before the experiment.

\section{III.A.4. Procedures}

Participants performed the experiment in a single 3-4 hour session. Individual tracking runs had a total length of $90 \mathrm{~s}$. The first $8.08 \mathrm{~s}$ of the run were considered the run-in time. This run-in time was included to allow the participants to stabilize the controlled dynamics and to adjust to the task. Data from the last $81.92 \mathrm{~s}$ were used as measurement data for data analysis.

Throughout the experiment, the eight experimental conditions were presented mixed and in a random order. This was done by performing sets of all eight conditions, for which the order was defined using a balanced Latin square. Breaks were held frequently to avoid fatigue and loss of motivation, typically after 2-3 sets of eight conditions.

For each condition, five repeated measurements at a constant level of tracking performance were collected as the measurement data. No fixed number of training runs was defined before the start of the experiment. The participants performed all conditions a minimum amount of eight times, where the experimenter kept track of their task performance. Additional measurements were collected if participants performance had not sufficiently stabilized within these eight measurements to collect the measurement data.

Tracking runs were started by the experimenter after a countdown from three. After completion of a $90 \mathrm{~s}$ tracking run, the participants received feedback of their task performance for the completed run, expressed in terms of the root mean square of the pitch and roll tracking error signals.

\section{III.A.5. Dependent Measures}

Several dependent measures were calculated from the measured control-loop signals for evaluating the effects of timevarying motion gains on tracking behavior. First, pilot performance and control activity in the roll and pitch axes of the target-following disturbance-rejection task were evaluated in terms of the time-domain variances of the error and control signals, respectively. The effects of the applied changes in motion gain are evaluated by comparing these 
signal variances over two 25 -second windows before and after the changes in motion gain occur (see Fig. 5). The error and control signal variances calculated over Window 2, as indicated in Fig. 5, are explicitly compared to those calculated over Window 1 by calculating their fraction. For example, the difference in the roll tracking error over these two windows is evaluated from the fraction $\sigma_{e_{\phi 2}}^{2} / \sigma_{e_{\phi 1}}^{2}$. Similar fractions are used for revealing changes in the pitch tracking error and control activity.

To explicitly evaluate changes in the adopted tracking behavior, the parameters of the time-varying pilot model introduced in Section II were estimated using a time-domain MLE method based on the approach described in Ref. 8. For every participant and condition the experimental data were averaged over the five collected measurement runs before the start of the estimation procedure. The control signal variance accounted for (VAF) was calculated as a measure for the accuracy of the pilot model in describing the measured control signal data for both the roll and pitch axes separately.

For reference, exactly the same procedure was followed for a time-invariant pilot model for which all pilot model parameters were assumed constant over the duration of the measurement. To evaluate the necessity for a time-varying model, the VAFs of both models will be compared for all conditions with time-varying motion gains. For the conditions with constant motion cueing gains $(\mathrm{C} 1$ and $\mathrm{C} 5)$, only this time-invariant model was fit to the data. In the following, these time-varying and time-invariant pilot models are referred to using the symbols $H_{p}(s, t)$ and $H_{p}(s)$, respectively.

Finally, the estimated pilot models were also used to assess the effects of the variations in motion cueing and tracking behavior on the open-loop dynamics for the pitch and roll axes. Changes in the characteristics of these openloop dynamics are expressed in terms of the crossover frequencies and phase margins of the target and disturbance open-loop systems ( $\omega_{c, t}$ and $\varphi_{m, t}$, and $\omega_{c, d}$ and $\varphi_{m, d}$, respectively) for both axes.

\section{III.B. Hypotheses}

For tracking tasks with controlled elements for which lead equalization is required, as those considered in the current study, it is known that motion feedback is typically used by pilots for reducing the amount of lead that needs to be generated from the visual display. ${ }^{2}$ Furthermore, the extent to which such motion feedback is used by pilots is affected by the quality of the supplied motion cues. Attenuation of the motion cues, either by scaling or high-pass filtering, has been shown to induce control with lower pilot gains and increased visual lead generation (higher $T_{L}$ ), which typically results in worse task performance. ${ }^{16,18}$

For the current experiment, where a time-varying reduction or increase in the gain of the supplied motion is considered, it is expected that similar changes in behavior will be observed for each individual axis. For a change in the motion filter gain in the pitch or roll axis, it is expected that a change in pilot tracking behavior for the corresponding axis will be visible. Conditions with reducing motion cueing gains are expected to show a decrease in pilot gains, an increase in visual lead time constants, and a reduction in task performance during the motion gain transition. For increasing motion filter gains, the opposite is expected: an increase in tracking performance, increase in pilot gains, and a reduction in visual lead equalization.

In addition to these direct effects of the applied motion gain variations on the tracking behavior in the corresponding axis, it is also anticipated that some cross-coupling between both axes will be apparent from the experiment data. For instance, a reduction in the motion gain in only one of the controlled axes, may not only affect tracking behavior in that axis, but also in the other controlled axis. Even though the cueing in that axis itself does not change, the overall motion sensation supplied to the pilot does, which is anticipated to yield behavioral adaptation. These changes in tracking behavior due to cross-coupling effects are expected to be equivalent to the direct effects of changes in motion gain, but smaller in magnitude.

\section{Results}

This section describes the results of the human-in-the-loop experiment. Measured tracking performance and control activity, parameter estimation results of the pilot model discussed in Section II.D, and crossover frequencies and phase margins are presented. An analysis of variance (ANOVA) was performed on the tracking performance and control activity data only, as these data are captured in a single parameter calculated for each run, allowing for a full factorial analysis. The pilot modeling results are obtained from two different models (see Section III.A.5), one for the time-invariant and one for the time-varying motion cueing conditions, and as such do not allow for a straightforward ANOVA analysis. 


\section{IV.A. Tracking Performance and Control Activity}

To quantify the difference in tracking performance and control activity due to the applied change in motion gain, the fractions of the tracking error and control input variances calculated over the initial and ending windows as defined in Section III.A.5 are presented in Fig. 6. The graphs in Fig. 6 show the relative change in $\sigma_{e_{\theta}}^{2}, \sigma_{e_{\phi}}^{2}, \sigma_{u_{\theta}}^{2}$, and $\sigma_{u_{\phi}}^{2}$ over the ending window (2) compared to the starting window (1). A fraction lower than 1.0 indicates better tracking performance or lower control activity after the transition in motion gain. Table 3 shows the results of three-way repeated-measures ANOVAs performed on the metrics of Fig. 6.

The gray lines in Fig. 6 indicate the tracking error and control signal variances for the conditions with constant motion cueing gains: $\mathrm{C} 1$ (solid gray line) and C5 (dashed gray line). If tracking performance and control activity were perfectly constant over the full length of the tracking run, both these lines should be at 1 in all four plots. As is clear from Fig. 6, even for these conditions with constant motion cueing, tracking performance was consistently better in the second window than in the first for pitch tracking, while worse performance over the second window is found for roll tracking. For both axes, control activity is seen to be consistently higher in the second measurement window, as can be verified from Fig. 6(c) and (d). These effects could be attributable to variations in the difficulty of the forcing functions over time.

When comparing performance and control activity before and after the evaluated motion gain transitions for the pitch task, better tracking performance and increased control activity are observed for the conditions with an increasing sigmoid in the pitch axis (C3 and $\mathrm{C} 4)$. For the conditions with a decreasing pitch sigmoid (C7 and C8), the opposite is observed: worse tracking performance and decreased control activity in the second measurement window. Overall, these opposite variations in $\sigma_{e_{\theta 2}}^{2} / \sigma_{e_{\theta 1}}^{2}$ and $\sigma_{u_{\theta 2}}^{2} / \sigma_{u_{\theta 1}}^{2}$ result in the statistically significant effects of SG and the significant interaction for $\mathrm{SG} * \mathrm{PS}$ listed in Table 3. The variations in the relative error variances shown in Fig. 6(a) are much more consistent (smaller variance bars) than those observed for $\sigma_{u_{\theta 2}}^{2} / \sigma_{u_{\theta 1}}^{2}$, hence only for the former the direct effect of the presence or absence of the pitch sigmoid (PS) is found to be statistically significant.

For the roll tracking task data, the observed variations in the relative error and control signal variances largely match the effects observed for the pitch tracking data. The conditions with an increasing roll sigmoid (C2 and $\mathrm{C} 4)$ show better tracking performance and increased control activity, while those with a decreasing sigmoid in the roll axis (C6 and C8) show the opposite effects. This variation over the experimental conditions again accounts for statistically significant effects of $\mathrm{SG}$ and the interaction $\mathrm{SG} * \mathrm{RS}$, as well as the main effect of RS for $\sigma_{e_{\phi 2}}^{2} / \sigma_{e_{\phi 1}}^{2}$. Fig. 6(d) further shows evidence for an interaction between the cueing and tracking in both axes. For the conditions with a constant roll cueing gain, but with increasing or decreasing sigmoids in the pitch axis (conditions $\mathrm{C} 3$ and C7), a decrease in control activity is also observed in the roll axis. This decrease in control activity is equivalent to that observed with the sigmoid in the pitch axis. As can be verified from Fig. 6(b), this decreased control activity, however, does not result in a corresponding change in tracking performance.

\section{IV.B. Variance Accounted For}

The VAF indicates the amount of variance in the measured pilot control signal accounted for by the pilot model. It is a measure of how well the model is able to describe the measured data. Fig. 7 and Fig. 8 provide the VAF for the pitch and roll axis, respectively. The figures show the mean values over all pilots and $95 \%$ confidence intervals corrected for between-subject variability. For conditions $\mathrm{C} 1$ and $\mathrm{C} 5$, only a time-invariant pilot model without time-varying parameters $\left(H_{p}(s)\right)$ is identified, as the motion filter gains remained constant over time in these conditions. This pilot model has 7 parameters in each axis to be estimated with MLE. For the remaining conditions the time-varying model $\left(H_{p}(s, t)\right)$ with 14 parameters in each axis, as discussed in Section II.D, is identified in addition to the time-invariant pilot model.

Fig. 7 shows that there is no significant difference in pitch control signal VAFs between the different conditions and between the two models. However, there seems to be a small decrease in VAF when the roll motion filter gain is increased over time ( $\mathrm{C} 2$ and $\mathrm{C} 4)$ and a small increase in VAF when the pitch motion filter gain is increased over time (C3 and C4). An opposite trend is visible when the motion gains are decreased over time (C6 - C8).

For the roll axis (Fig. 8), opposite trends can be observed compared to the pitch axis. The VAF in the roll axis is significantly lower than the VAF in the pitch axis. This indicates that pilots behaved more linearly in pitch compared to roll. Finally, it can be observed that for all conditions with time-varying motion gains, the pilot model with timevarying parameters, $H_{p}(s, t)$, has a slightly higher VAF compared to the constant pilot model, $H_{p}(s)$. This indicates that the time-varying model is able to better describe the measured data in these conditions, albeit marginally. 
(a) Error variance, pitch

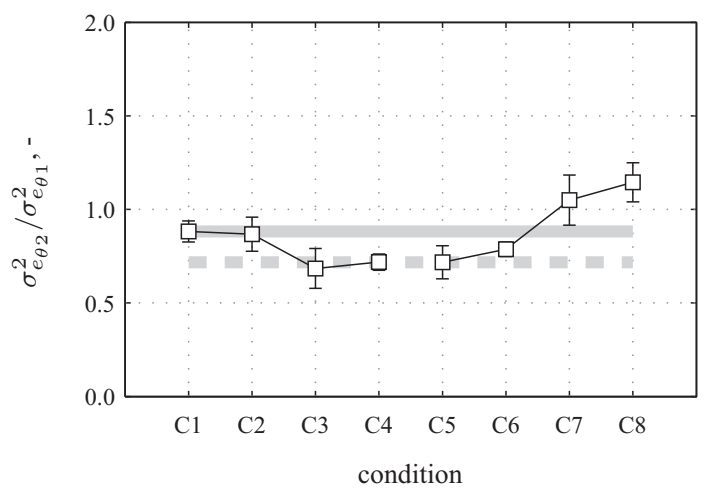

(c) Control variance, pitch

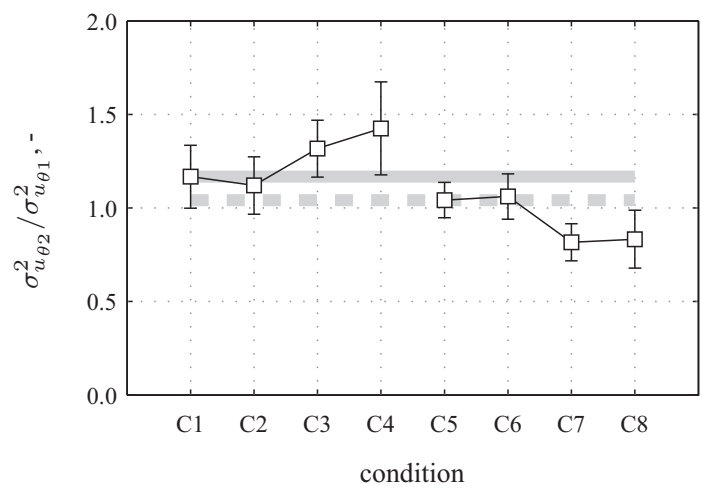

(b) Error variance, roll

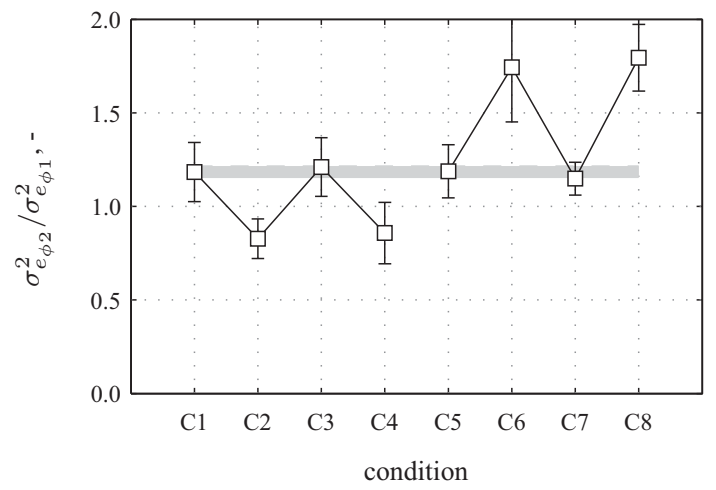

(d) Control variance, roll

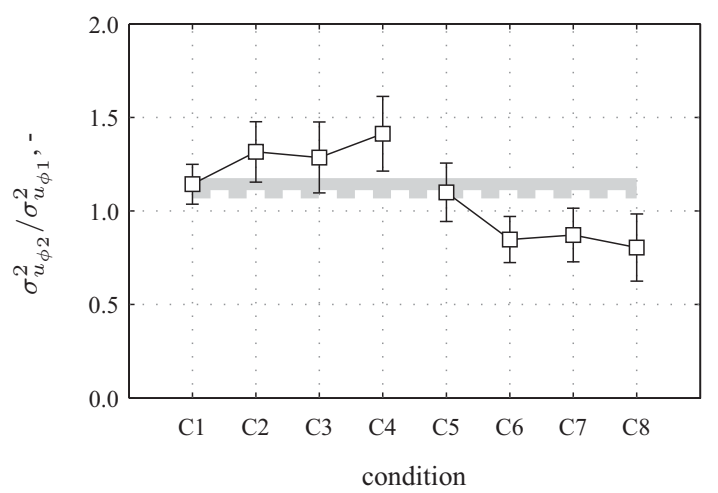

Figure 6. Relative windowed tracking error and control input variances for pitch and roll tracking.

Table 3. Three-way repeated-measures ANOVA results for the relative windowed pitch and roll tracking error and control signal variances, where $* *$ is significant $(p<0.05)$ and - is not significant $(p \geq 0.05)$.

\begin{tabular}{lrrrrrrrr}
\hline \hline \multirow{2}{*}{ Effect } & \multicolumn{8}{c}{ Dependent measures } \\
\cline { 2 - 9 } & \multicolumn{1}{c}{$\sigma_{e_{\theta_{2}}^{2}}^{2} / \sigma_{e_{\theta_{1}}}^{2}$} & \multicolumn{1}{c}{$\sigma_{e_{\phi_{2}}}^{2} / \sigma_{e_{\phi_{1}}}^{2}$} & \multicolumn{1}{c}{$\sigma_{u_{\theta_{2}}}^{2} / \sigma_{u_{\theta_{1}}}^{2}$} & \multicolumn{2}{c}{$\sigma_{u_{\phi_{2}}}^{2} / \sigma_{u_{\phi_{1}}}^{2}$} \\
\cline { 2 - 9 } & $\mathrm{F}$ & Sig. & \multicolumn{1}{c}{$\mathrm{F}$} & Sig. & \multicolumn{1}{c}{$\mathrm{F}$} & Sig. & \multicolumn{1}{c}{$\mathrm{F}$} & Sig. \\
\hline SG & 35.95 & $* *$ & 23.80 & $* *$ & 23.30 & $* *$ & 23.50 & $* *$ \\
$\mathrm{PS}$ & 7.63 & $* *$ & 0.31 & - & 0.00 & - & 0.07 & - \\
$\mathrm{RS}$ & 4.90 & - & 17.41 & $* *$ & 0.24 & - & 0.01 & - \\
$\mathrm{SG} * \mathrm{PS}$ & 89.12 & $* *$ & 0.11 & - & 12.13 & $* *$ & 3.36 & - \\
$\mathrm{SG} * \mathrm{RS}$ & 1.54 & - & 92.26 & $* *$ & 0.01 & - & 6.42 & $* *$ \\
$\mathrm{PS} * \mathrm{RS}$ & 0.34 & - & 0.12 & - & 1.38 & - & 4.01 & - \\
$\mathrm{SG} * \mathrm{PS} * \mathrm{RS}$ & 0.03 & - & 0.20 & - & 1.95 & - & 3.49 & - \\
\hline \hline
\end{tabular}

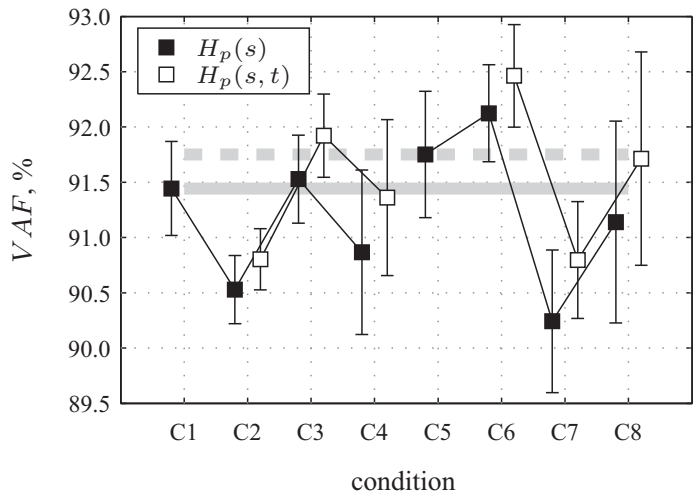

Figure 7. Pitch control signal variance accounted for.

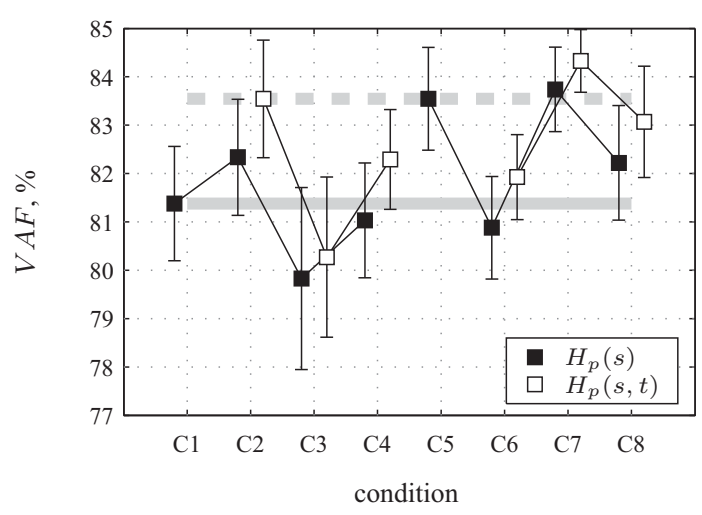

Figure 8. Roll control signal variance accounted for. 


\section{IV.C. Pilot Control Behavior}

The estimated pilot model parameters for the pitch axis are given in Fig. 9. Fig. 10 depicts the same parameters for the roll axis. Finally, Fig. 11 depicts the time-of-maximum-rate and maximum-rate parameters for the pitch and roll axes. Note that the presented parameter estimates were obtained from the time-invariant pilot model $H_{p}(s)$ for conditions $\mathrm{C} 1$ and $\mathrm{C} 5$, and from $H_{p}(s, t)$ for all other conditions. All plots show means over all subjects and $95 \%$ confidence intervals corrected for between-subject variability.

Fig. 9a shows the pilot visual gain in the pitch axis. A significant increase in visual gain can be observed from $K_{v 1}$ to $K_{v 2}$ for conditions with an increasing pitch motion filter gain (C3 and C4). A less significant opposite trend can be seen for conditions with a decreasing pitch motion filter gain (C7 and C8). Note that the gray horizontal lines represent the data from $\mathrm{C} 1$ and $\mathrm{C} 5$; that is, the data for the conditions with a constant low or a high motion filter gain, respectively. For the lead time constant depicted in Fig. $9 \mathrm{~b}$ an opposite trend can be observed. For conditions where the pitch motion gain increases over time, the lead time constant decreases and vice versa. The pilot motion gain (Fig. 9c) shows a similar trend to the pilot visual gain but less significant. The observed effects in pilot equalization $\left(K_{v}, T_{l}\right.$, and $\left.K_{m}\right)$ indicate an increased use of motion cues when the motion filter gain is increased over time. No significant cross-coupling effects in equalization dynamics are observed; that is, for a change in roll motion filter gain only ( $\mathrm{C} 2$ and $\mathrm{C} 6)$, the equalization dynamics in pitch are equivalent to the dynamics in the conditions with constant motion filter gains ( $\mathrm{C} 1$ and $\mathrm{C} 5)$ and remain constant.

The pilot visual and motion time delays in the pitch axis (Figures $9 \mathrm{~d}$ and 9e) are relatively constant over all conditions. Only small variations can be observed in the order of $10 \mathrm{~ms}$. The pilot motion time delay is significantly lower than the visual time delay. Finally, the neuromuscular damping and frequency do not show any significant variations over the conditions (Figures $9 \mathrm{f}$ and $9 \mathrm{~g}$ ). However, a small drop in neuromuscular damping and frequency might be present for conditions with a decreasing pitch motion sigmoid (C7 and C8).

Pilot equalization in the roll axis is much less affected by the time-varying motion filter gains compared to equalization in the pitch axis. This is to be expected considering the pilot performance and control activity, and VAF results discussed in the previous sections. Fig. 10a shows a slight increase of visual gain when the roll motion filter gain increases ( $\mathrm{C} 2$ and $\mathrm{C} 4)$. However, also an increase can be observed for the condition with only an increase in pitch motion filter gain (C3). This might indicate cross-coupling in behavior between the roll and pitch axes. An opposite trend can be seen for the conditions with a decreasing motion filter gain. The visual lead time constant in the roll axis (Fig. 10b) shows an opposite trend to $K_{v}$, including the cross-coupling seen for condition C3. Similar to the pitch axis, Fig. 10c shows less significant effects in pilot motion gain compared to pilot visual gain. In addition, a cross-coupling effect is not visible for this parameter. Overall, these trends in the pilot equalization parameters in the roll axis suggest an increased use of simulator motion cues for an increase in motion filter gain, even when the increase in motion filter gain is in the pitch axis.

Figures $10 \mathrm{~d}$ and $10 \mathrm{e}$ show that there are minimal effects to the pilot visual and motion time delays in the roll axis, similar to the pitch axis. The pilot motion time delay is about half the magnitude of the visual time delay. Furthermore, similar to the pitch axis, no significant effects are observed for the neuromuscular damping and frequency (Figures 10f and $10 \mathrm{~g})$.

Comparing the parameters for pitch and roll (Fig. 9 with Fig. 10), it can be observed that pilot visual and motion gains, and lead time constants are lower in the roll axis. In addition, pilot visual and motion time delays are higher. This is consistent with the lower tracking performance observed for roll tracking compared to pitch tracking.

Fig. 11a shows no significant variations for the time of maximum rate of change $M$ in roll and pitch. The time of maximum rate of change for the motion filter gains was equal to $49.04 \mathrm{~s}$. In both the roll and pitch pilot model, the estimated value for $M$ is around $50 \mathrm{~s}$, just after the change in motion filter gain. Furthermore, no significant variations are found for the maximum rate of change $G$ in both axes (Fig. 11b). The maximum rate of change is, however, found to be higher than the value of 0.5 used for the motion filter gains. This suggests that pilots adapt their behavior more abruptly to the change in simulator motion compared to the actual rate of change of the motion.

\section{IV.D. Open-Loop Characteristics}

Using the transfer functions of the identified pilot models and the controlled dynamics, the (time-varying) disturbance and target open-loop responses were calculated. ${ }^{26}$ Figures 12 and 13 depict the corresponding crossover frequencies and phase margins for the pitch and roll axis, respectively.

Fig. 12a indicates that the disturbance crossover frequency in the pitch axis increases from the initial value $\omega_{c 1}$ to the final value $\omega_{c 2}$ for the conditions with an increasing pitch motion sigmoid (C3 and $\left.\mathrm{C} 4\right)$. Fig. 12c shows a corresponding drop in disturbance phase margin from the initial value $\varphi_{m 1}$ to the final value $\varphi_{m 2}$ for these conditions. 
(a) Visual gain

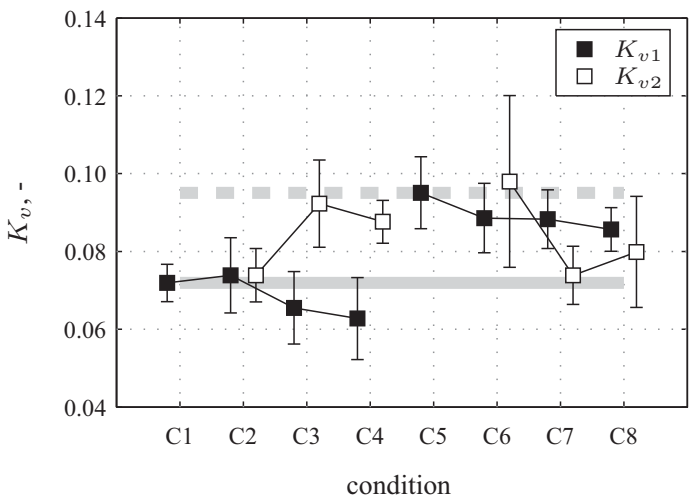

(b) Lead time constant

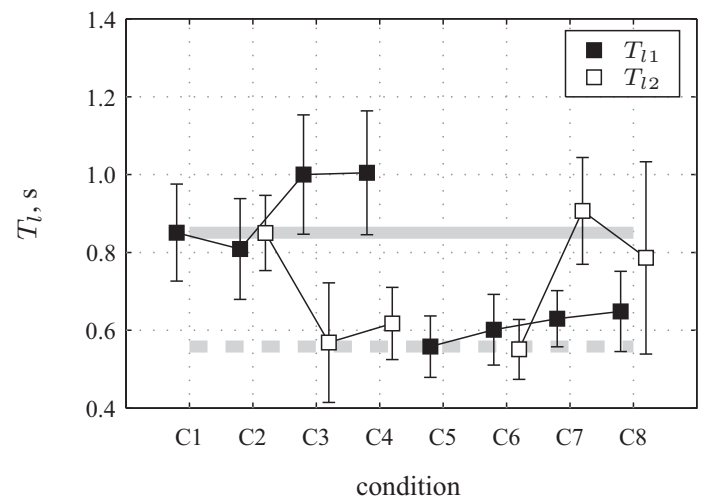

(c) Vestibular gain

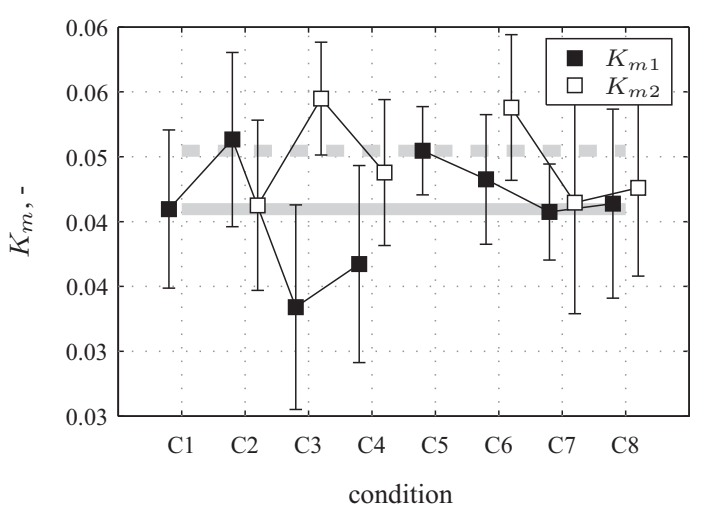

(d) Visual delay

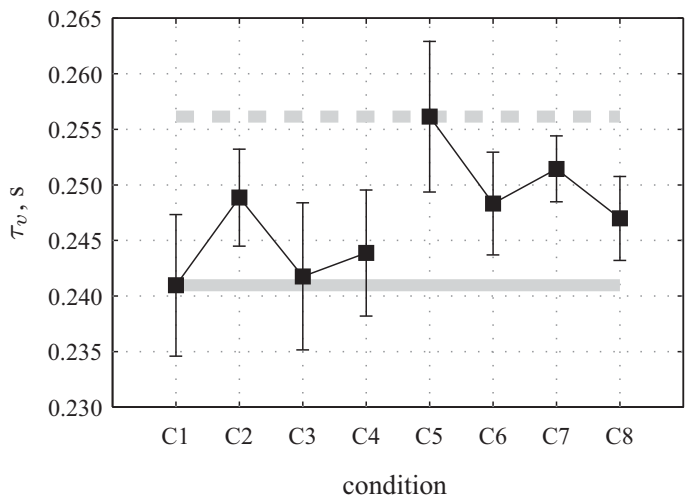

(f) Neuromuscular damping

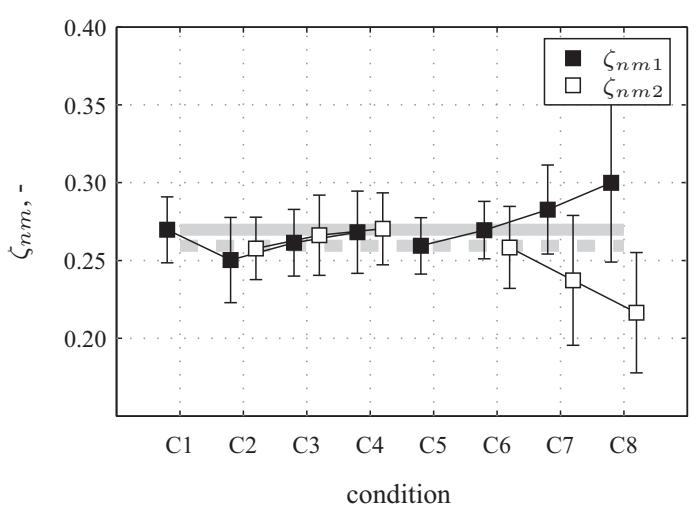

(e) Motion delay

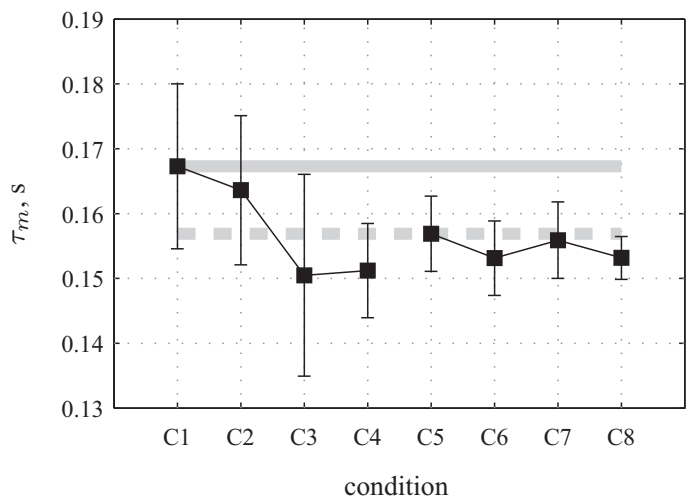

(g) Neuromuscular frequency

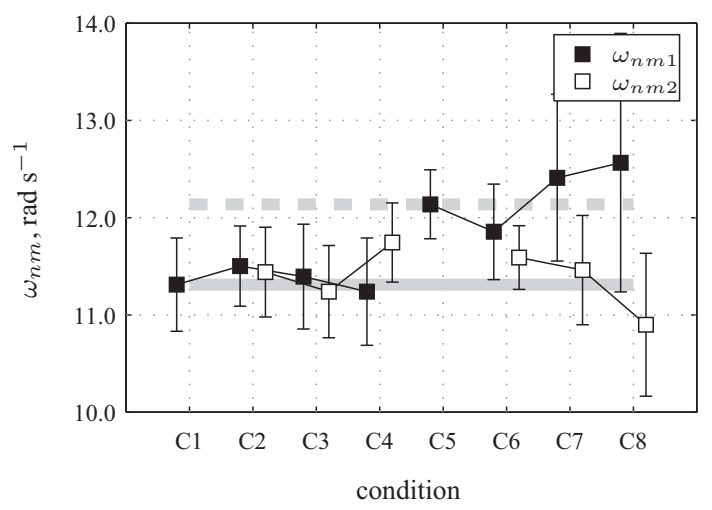

Figure 9. Pilot model parameters in the pitch axis. 
(a) Visual gain

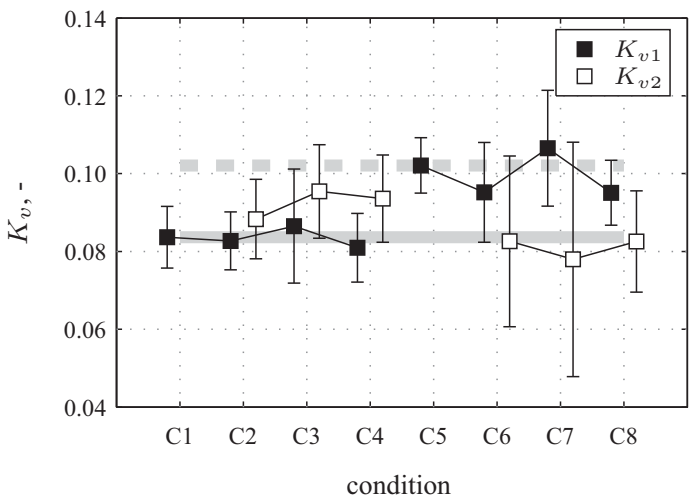

(b) Lead time constant

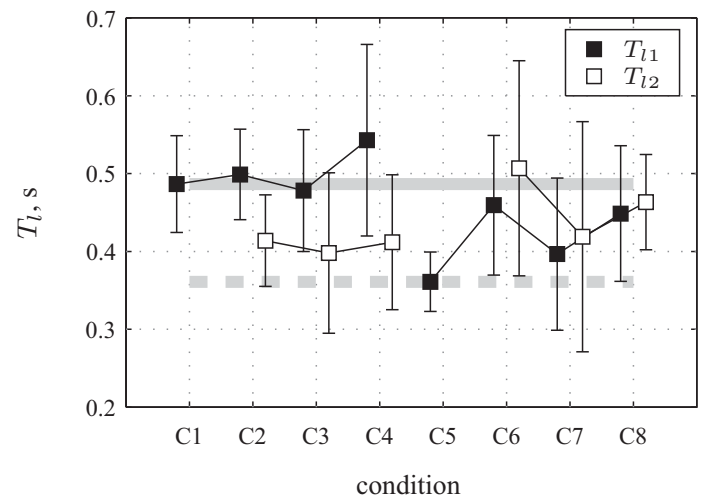

(c) Vestibular gain

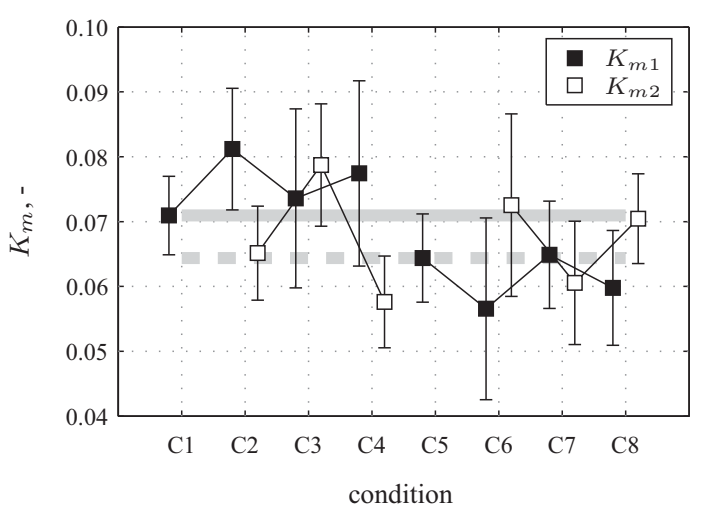

(d) Visual delay

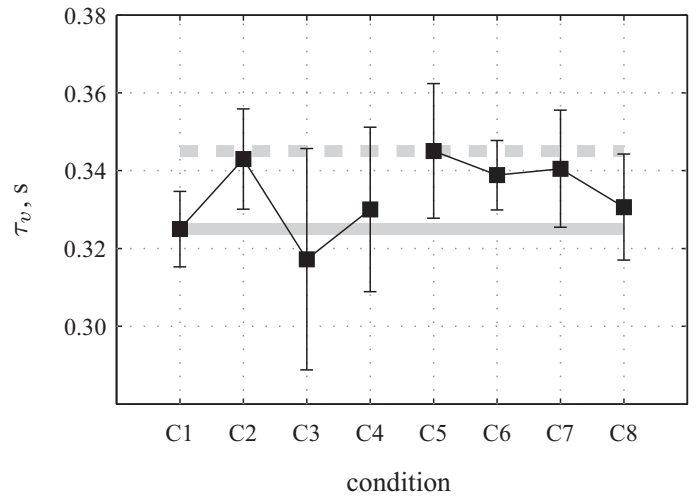

(f) Neuromuscular damping

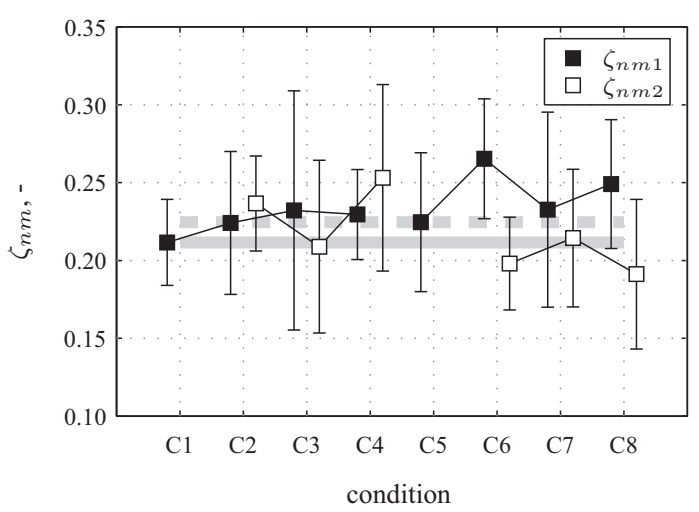

(e) Motion delay

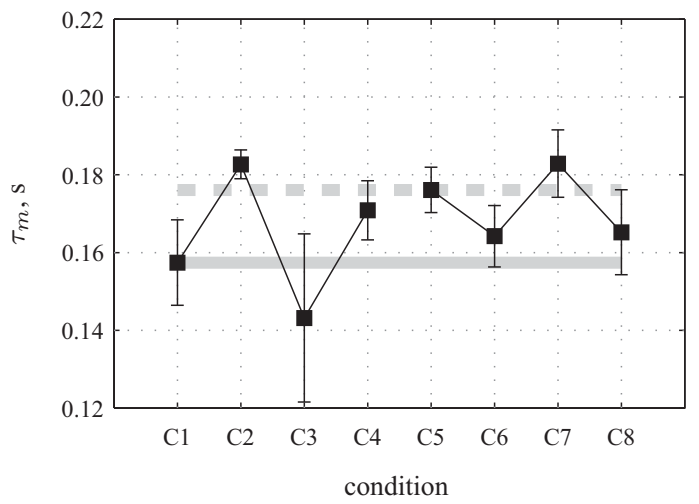

(g) Neuromuscular frequency

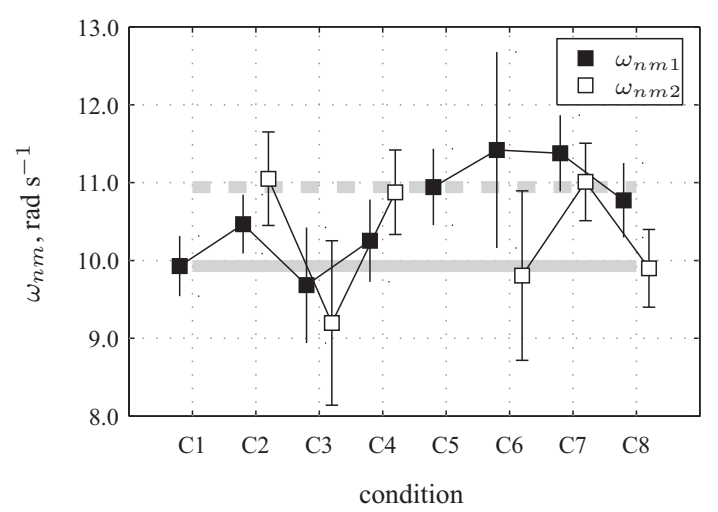

Figure 10. Pilot model parameters in the roll axis. 
(a) Time of maximum growth

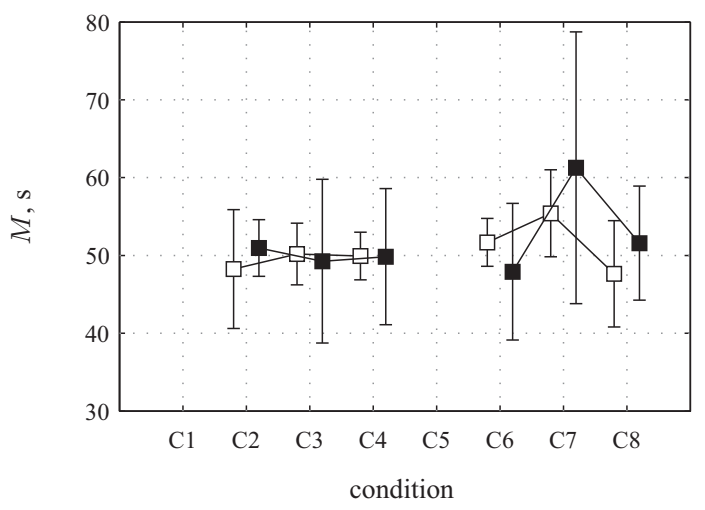

(b) Growth rate

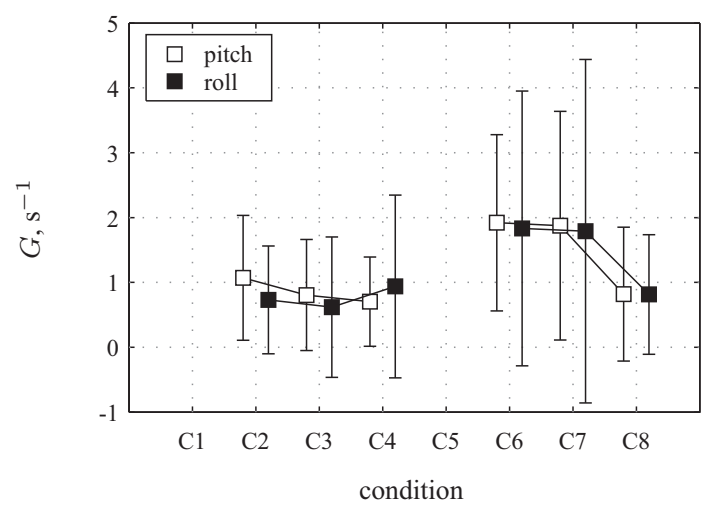

Figure 11. Pilot model time-variation parameters.

These trends are consistent with previous research and indicate an increase in disturbance-rejection performance at the cost of a small drop in stability margins with increasing simulator motion cueing gains. Opposite trends can be observed for the conditions with a decreasing pitch motion sigmoid (C7 and C8). For the target crossover frequencies and phase margins in pitch (Figures 12b and 12d ) opposite trends are visible compared to the disturbance crossover frequencies and phase margins. With an increase in pitch motion gain, target-following performance drops while stability margins increase, and vice versa for a decrease in pitch motion cueing gain.

The trends in crossover frequencies and phase margins for a change in roll motion gain in the roll axis are similar to the trends for a change in pitch motion gain in the pitch axis. Fig. 13a indicates an increase in disturbance crossover frequency for conditions $\mathrm{C} 2$ and $\mathrm{C} 4$. The disturbance crossover frequency decreases for the conditions with a decreasing roll motion sigmoid. The disturbance phase margins are not significantly affected and remain approximately constant for all conditions (Fig. 13c). For the target open-loop in the roll axis, a decrease in crossover frequency and an increase in phase margin can be observed for the conditions with an increasing roll motion gain (Figures 13b and 13d). Opposite trends are visible for the conditions with a decreasing roll motion gain.

Comparing the results in the pitch and roll axes, significantly higher disturbance and target crossover frequencies are observed in pitch. The disturbance phase margin is also markedly higher in pitch, while the target phase margin is at similar levels in both axes. These crossover frequency and phase margin results are consistent with the performance results presented in Section IV.A, which also indicate an increase in roll or pitch tracking performance when motion gains are increased in the corresponding axis, and higher performance in pitch compared to roll.

\section{Discussion}

An experiment was conducted on a motion-base simulator in which pilots performed a simultaneous roll and pitch tracking task. In different experimental conditions, the roll and/or pitch motion cueing gains were varied over time, analogous to the functioning of adaptive motion filters. The controlled dynamics and forcing function characteristics were equivalent in both axes of control.

Pitch and roll tracking performance and control activity were found to increase over time when motion filter gains increased in the same axis. Opposite trends were found for a time-varying decrease in motion gain. The variations in performance were apparent from the calculated tracking error variances, as well as from the measured crossover frequencies and phase margins. From estimated pilot model parameters, it was found that the change in performance was a result of a change in the pilot equalization dynamics caused by the changing motion feedback. With increasing motion gains, pilot visual and motion gains increased and visual lead time constants decreased in both axes. Opposite trends are found for a time-varying decrease of the motion gains. Pilot delays and neuromuscular dynamics were not significantly affected by the change in motion gains. Some pilot model parameters in roll show a modest crosscoupling effect when the motion gain is varied in pitch: changes in roll-axis pilot gains and control activity were found for conditions in which only the pitch motion gain was changed. Such cross-coupling in behavior between control axes was also observed in a previous study with a multi-axis control task. ${ }^{8}$

Although overall observations were equivalent in pitch and roll, the observed behavioral variations were much less strong in roll, even though the controlled dynamics and forcing function properties were identical in both axes. In 
(a) Pitch disturbance crossover frequency

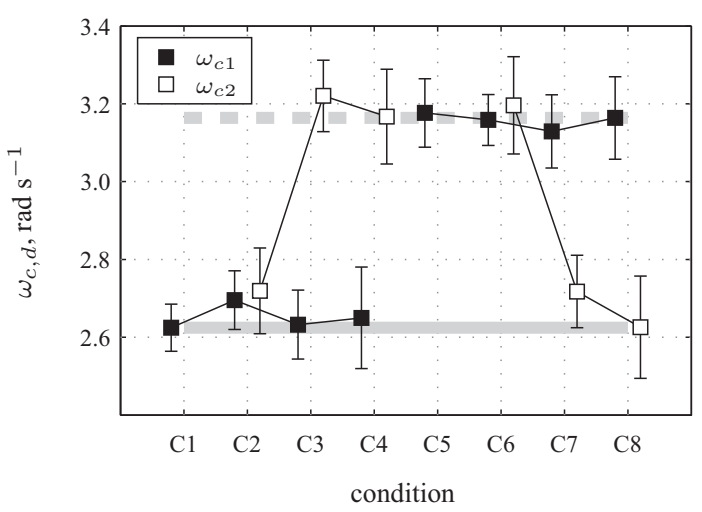

(c) Pitch disturbance phase margin

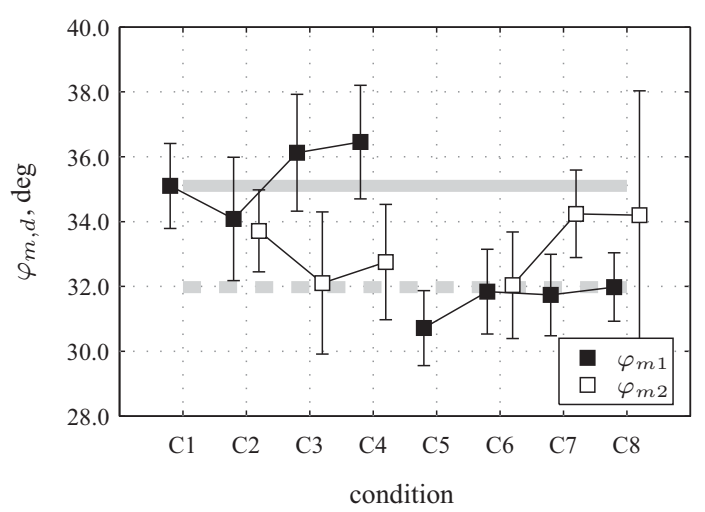

(b) Pitch target crossover frequency

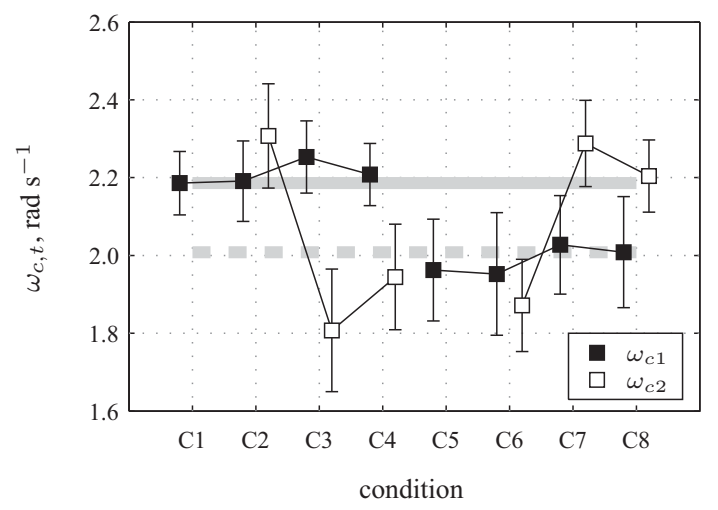

(d) Pitch target phase margin

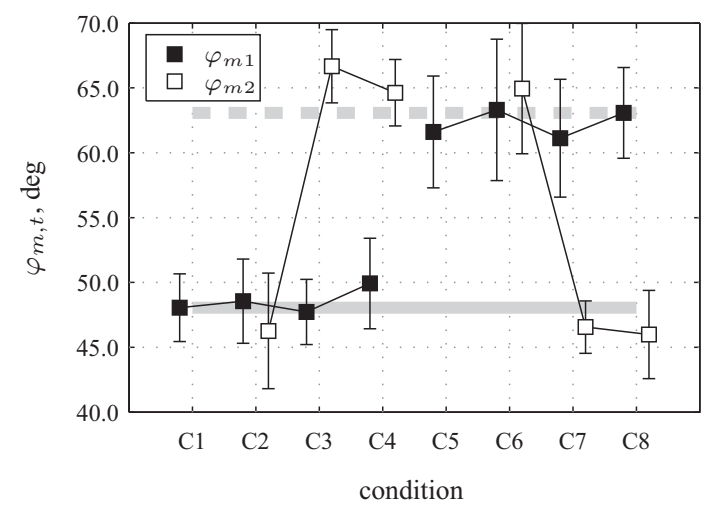

Figure 12. Pitch-axis crossover frequencies and phase margins.

addition, overall performance was much lower in the roll axis. This might be caused by the different presentation of roll and pitch errors on the compensatory display. Roll errors were presented by an angular difference between the aircraft symbol and the horizon line, while pitch errors were presented by a vertical distance between the aircraft symbol and the horizon line. Alternatively, the differences in roll and pitch tracking performance could also be the result of the different control inceptor and inherent neuromuscular actuation dynamics in both axis. Most likely, however, is that the observed difference in performance is simply caused by the control strategy selected by the pilots. This suggests that they may have put more emphasis on reducing pitch errors than roll errors. Pilots were not instructed to adopt a certain control strategy, but some indicated that they gave pitch error reduction a higher priority. A number of pilots indicated that they adopted a control strategy with intermittent control of roll and pitch. This is common when attention needs to be divided between to different tasks. This intermittent control was, however, not found to have significant effects on the ability to accurately identify pilot models using the measured time-domain signals. Higher pilot remnant levels and as a consequence lower VAFs were, however, found in this study compared to previous research with comparable single-axis control tasks. ${ }^{20,24}$

In addition to the time variations in pilot performance and control activity in the conditions with time-varying motion gains, differences over time were also found in the conditions with constant motion gains ( $\mathrm{C} 1$ and $\mathrm{C} 5)$. This might be the result of variations in difficulty in compensating for the target and disturbance forcing functions over time. These variations are inherently related to the selected phase settings of the sinusoids in each forcing function. For the selection of these phases for the multisine forcing functions used in the experiment, no explicit care was taken to equalize the properties of these signals over the length of the tracking runs.

Conditions with increasing and decreasing motion gains were evaluated in the experiment. Adaptation of pilot behavior to the increasing or decreasing motion gains was found to be somewhat asymmetric. For example, a larger increase in pilot visual gain is present for an increase in pitch motion filter gain than the decrease in pilot visual gain that is observed for a decrease in pitch motion filter gain. Such asymmetry is also visible in other parameters. This might be caused by the fact that pilots are startled by a sudden increase in motion intensity, prompting them to use the motion feedback more efficiently to minimize the disturbance. This surprise effect is not present when the motion 
(a) Roll disturbance crossover frequency

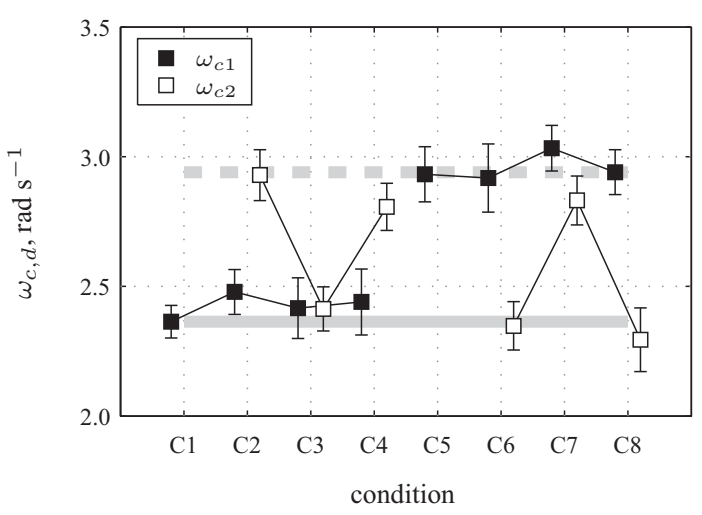

(c) Roll disturbance phase margin

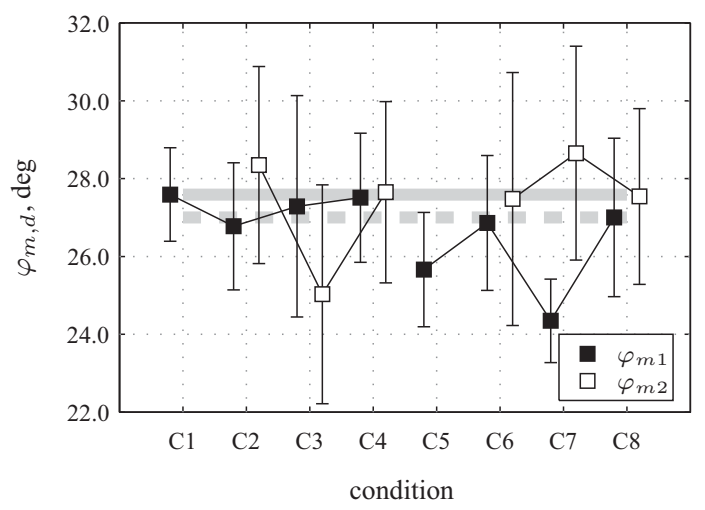

(b) Roll target crossover frequency

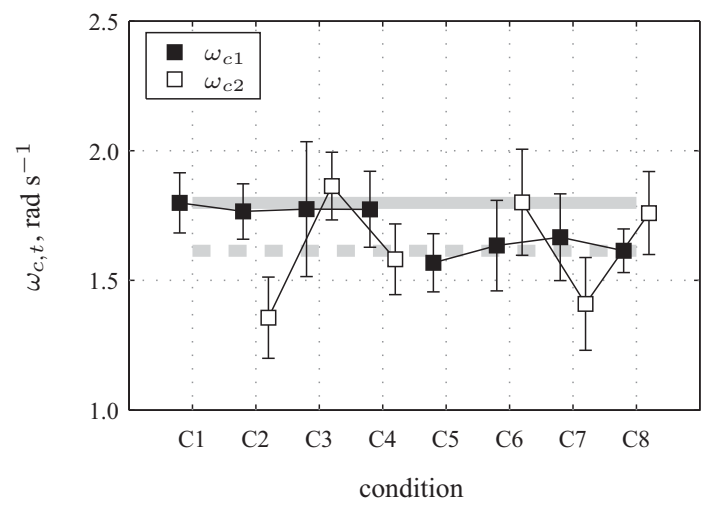

(d) Roll target phase margin

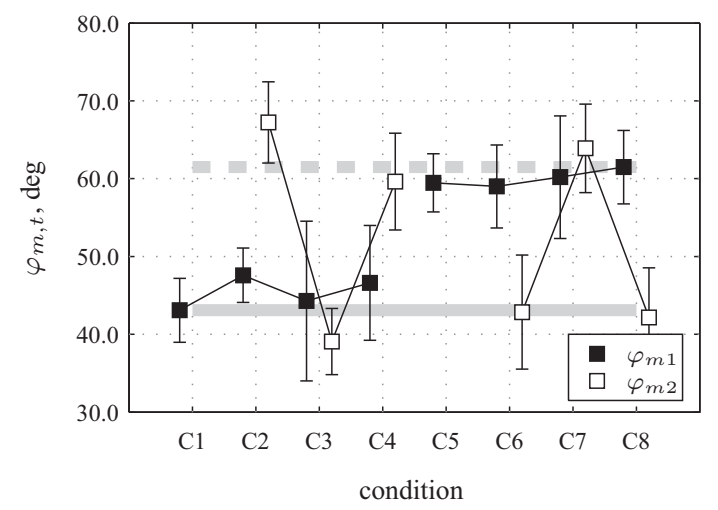

Figure 13. Roll-axis crossover frequencies and phase margins.

intensity is reduced. Another explanation could be that starting the task with a high motion filter gain, allows pilots to better tune their internal model to the task. This internal model can then be used to increase performance after the motion gain is reduced (compared to the performance when starting with a low motion gain).

Finally, for the conditions with time-varying motion gains the differences in VAF between the constant and timevarying pilot models are found to be small. This warrants the question if the use of a time-varying pilot model, which has 7 additional model parameters, is appropriate to model pilot control behavior in these conditions. Despite the marginal increase in VAF, significant and consistent variations were found in the time-varying parameters of the pilot model. This indicates that the parameter estimation technique using time-varying sigmoid functions is able to capture small variations in pilot behavior accurately, despite the small effect on the total pilot model output. However, due to the use of an explicit function for modeling the time variation (sigmoid), the method is limited to model only a single increase or decrease in parameters, and prior knowledge is required about the time-varying nature of the control task.

\section{Conclusions}

A pilot model with time-varying parameters was successfully used to model pilot adaptation to time-varying motion filter gains in a multi-axis pitch and roll tracking task. For an increase in motion filter gain in roll or pitch, pilot equalization adapted in the same axis as the motion gain variation, as was indicated by an increase in pilot visual and motion gains and a decrease in visual lead time constant. This allowed pilots to increase tracking performance in that particular axis as the motion gain increased. Opposite trends were found for a time-varying decrease in motion gain. Although these observed behavioral changes were similar in roll and pitch, they were less strong in roll. In addition, performance in attenuating pitch errors was much better compared to attenuating roll errors. Overall, these main observed changes in tracking performance and control behavior match those found for single-axis tracking tasks with different static motion cueing gains in earlier experiments. Finally, the current experiment also shows indications of minor cross-coupling effects in behavior between pitch and roll, as small variations in roll tracking were observed when only changes in pitch motion cueing were present. 


\section{References}

${ }^{1}$ McRuer, D. T. and Jex, H. R., "A Review of Quasi-Linear Pilot Models," IEEE Transactions on Human Factors in Electronics, Vol. HFE-8, No. 3, 1967, pp. 231-249.

${ }^{2}$ Shirley, R. S. and Young, L. R., "Motion Cues in Man-Vehicle Control," Fourth Annual NASA-University Conference on Manual Control, University of Michigan, Ann Arbor (MI), 21-23 March 1968, pp. 435-448.

${ }^{3}$ Schroeder, J. A. and Grant, P. R., "Pilot Behavioral Observations in Motion Flight Simulation," Proceedings of the AIAA Guidance, Navigation, and Control Conference and Exhibit, Toronto (ON), Canada, No. AIAA-2010-8353, 2-5 Aug. 2010.

${ }^{4}$ Young, L. R., "On Adaptive Manual Control," IEEE Transactions on Man-Machine Systems, Vol. 10, No. 4, Dec. 1969, pp. $292-331$.

${ }^{5}$ Thompson, P. M., Klyde, D. H., and Brenner, M. J., "Wavelet-Based Time-Varying Human Operator Models," Proceedings of the AIAA Atmospheric Flight Mechanics Conference and Exhibit, Montreal (CA), No. AIAA-2001-4009, 6-9 Aug. 2001.

${ }^{6}$ Zaal, P. M. T. and Sweet, B. T., "Estimation of Time-Varying Pilot Model Parameters," Proceedings of the AIAA Modeling and Simulation Technologies Conference, Portland (OR), No. AIAA-2011-6474, 8-11 Aug. 2011.

${ }^{7}$ Hess, R. A., "A Preliminary Study of Human Pilot Dynamics in the Control of Time-Varying Systems," Proceedings of the AIAA Modeling and Simulation Technologies Conference, Portland (OR), No. AIAA-2011-6554, 8-11 Aug. 2011.

${ }^{8}$ Zaal, P. M. T. and Sweet, B. T., "Identification of Time-Varying Pilot Control Behavior in Multi-Axis Control Tasks," Proceedings of the AIAA Modeling and Simulation Technologies Conference, Minneapolis (MN), No. AIAA-2012-4793, 13-16 Aug. 2012. 2104

${ }^{9}$ Hess, R. A., "Modeling Pilot Detection of Time-Varying Aircraft Dynamics," Journal of Aircraft, Vol. 49, No. 6, Nov.-Dec. 2012, pp. 2100-

${ }^{10}$ Parrish, R. V., Dieudonne, J. E., Bowles, R. L., and Martin, Jr., D. J., "Coordinated Adaptive Washout for Motion Simulators," Journal of Aircraft, Vol. 12, No. 1, 1975, pp. 44-50.

${ }^{11}$ Ariel, D. and Sivan, R., "False Cue Reduction in Moving Flight Simulators," IEEE Transactions on Systems, Man, and Cybernetics, Vol. SMC-14, No. 4, July-Aug. 1984, pp. 665-671.

${ }^{12}$ Nahon, M. A., Reid, L. D., and Kirdeikis, J., "Adaptive Simulator Motion Software with Supervisory Control," Journal of Guidance, Control, and Dynamics, Vol. 15, No. 2, March-April 1992, pp. 376-383.

${ }^{13}$ Telban, R. J., Wu, W., and Cardullo, F. M., "Motion Cueing Algorithm Development: Initial Investigation and Redesign of the Algorithms," Tech. Rep. NASA CR-2000-209863, Department of MechanicaI Engineering, State University of New York, Binghamton, New York, 2000.

${ }^{14}$ Naseri, A. and Grant, P. R., "An Improved Adaptive Motion Drive Algorithm," Proceedings of the AIAA Modeling and Simulation Technologies Conference and Exhibit, San Francisco (CA), No. AIAA-2005-6500, 15-18 Aug. 2005.

${ }^{15}$ Hwang, T.-S., Kuo, M.-S., and Hsieh, S.-P., "Optimal Genetic and Adaptive Fuzzy Washout Filter Design in the Motion-Cueing Simulator," Asian Journal of Control, Vol. 10, No. 1, January 2008, pp. 88-95.

${ }^{16}$ Pool, D. M., Damveld, H. J., van Paassen, M. M., and Mulder, M., "Tuning Models of Pilot Tracking Behavior for a Specific Simulator Motion Cueing Setting," Proceedings of the AIAA Modeling and Simulation Technologies Conference, Portland (OR), No. AIAA-2011-6322, 8-11 Aug. 2011.

${ }^{17}$ Zaal, P. M. T., Pool, D. M., van Paassen, M. M., and Mulder, M., "Comparing Multimodal Pilot Pitch Control Behavior Between Simulated and Real Flight," Journal of Guidance, Control, and Dynamics, Vol. 35, No. 5, Sept. - Oct. 2012, pp. 1456-1471.

${ }^{18}$ Pool, D. M., Zaal, P. M. T., Damveld, H. J., and van Paassen, M. M. M. M., "Evaluating Simulator Motion Fidelity using In-Flight and Simulator Measurements of Roll Tracking Behavior," Proceedings of the AIAA Modeling and Simulation Technologies Conference, Minneapolis $(M N)$, No. AIAA-2012-4635, 13-16 Aug. 2012.

${ }^{19}$ Stapleford, R. L., Peters, R. A., and Alex, F. R., "Experiments and a Model for Pilot Dynamics with Visual and Motion Inputs," Tech. Rep. NASA CR-1325, NASA, 1969.

${ }^{20}$ Zaal, P. M. T., Pool, D. M., Mulder, M., and van Paassen, M. M., "Multimodal Pilot Control Behavior in Combined Target-Following Disturbance-Rejection Tasks," Journal of Guidance, Control, and Dynamics, Vol. 32, No. 5, Sept.-Oct. 2009, pp. 1418-1428.

${ }^{21}$ McRuer, D. T., Graham, D., Krendel, E. S., and Reisener, W., "Human Pilot Dynamics in Compensatory Systems. Theory, Models and Experiments With Controlled Element and Forcing Function Variations," Tech. Rep. AFFDL-TR-65-15, Wright Patterson AFB (OH): Air Force Flight Dynamics Laboratory, 1965.

${ }^{22}$ Shirley, R. S. and Young, L. R., “Motion Cues in Man-Vehicle Control," IEEE Transactions on Man Machine Systems, Vol. 9, No. 4, Dec. 1968, pp. 121-128.

${ }^{23}$ Zaal, P. M. T., Pool, D. M., de Bruin, J., Mulder, M., and van Paassen, M. M., "Use of Pitch and Heave Motion Cues in a Pitch Control Task," Journal of Guidance, Control, and Dynamics, Vol. 32, No. 2, March-April 2009, pp. 366-377.

${ }^{24}$ Pool, D. M., Zaal, P. M. T., van Paassen, M. M., and Mulder, M., "Effects of Heave Washout Settings in Aircraft Pitch Disturbance Rejection," Journal of Guidance, Control, and Dynamics, Vol. 33, No. 1, Jan.-Feb. 2010, pp. 29-41.

${ }^{25}$ Damveld, H. J., Beerens, G. C., van Paassen, M. M., and Mulder, M., "Design of Forcing Functions for the Identification of Human Control Behavior," Journal of Guidance, Control, and Dynamics, Vol. 33, No. 4, July - Aug. 2010, pp. 1064-1081.

${ }^{26}$ Jex, H. R. and Magdaleno, R. E., "Roll Tracking Effects of G-vector Tilt and Various Types of Motion Washout," Fourteenth Annual Conference on Manual Control, University of Southern California, Los Angeles (CA), 25-27 April 1978, pp. 463-502.

${ }^{27}$ Berkouwer, W. R., Stroosma, O., van Paassen, M. M., Mulder, M., and Mulder, J. A., "Measuring the Performance of the SIMONA Research Simulator's Motion System," Proceedings of the AIAA Modeling and Simulation Technologies Conference and Exhibit, San Francisco (CA), No. AIAA-2005-6504, 15-18 Aug. 2005.

${ }^{28}$ Stroosma, O., van Paassen, M. M., Mulder, M., and Postema, F. N., "Measuring Time Delays in Simulator Displays," Proceedings of the AIAA Modeling and Simulation Technologies Conference and Exhibit, Hilton Head (SC), No. AIAA-2007-6562, 20-23 Aug. 2007. 